\title{
Müller, Philipp: Geschichte machen. Historisches Forschen und die Politik der Archive, 517 S., Wallstein, Göttingen 2019.
}

\author{
Katrin Minner
}

Angenommen: 29. April 2021 / Online publiziert: 22. Mai 2021

(C) Der/die Autor(en) 2021

Liest man/frau die Studie von Philipp Müller zum Spannungsfeld von historischer Forschung und Archivpolitik im 19. Jahrhundert, so fühlt sich die Leserin an erstaunlich virulente Gegebenheiten der Archivbenutzung noch im 20. Jahrhundert, zum Teil bis heute, erinnert: die Relevanz von Empfehlungsschreiben, die Beantragung von Genehmigungen zur Einsichtnahme in gesperrte Bestände und die mit rechtlichen Argumenten beschränkte Herausgabe beziehungsweise Verwendung von Akten - und das in einer Ära von mittlerweile festgeschriebenem Jedermann/-frauund Informationsfreiheitsgesetz. Auch heute sind Forscher_innen häufig auf das Wissen der Archivar_innen angewiesen und dass diese ihnen Informationen zugänglich machen. Als Gatekeeper üben die Archivmitarbeiter_innen weiterhin die Kontrolle über Findmittel und Unterlagen aus. Ein langer Schatten des vormodernen Archivwesens?

In der Studie kristallisiert sich eine bemerkenswerte Kontinuität von frühneuzeitlich geprägten Denkweisen und Praktiken seitens der für die Archive zuständigen Beamten, Verwaltungen und Ministerien heraus - und bricht dadurch mit der bisherigen Historiografie zur Archivpolitik: Sie relativiert die bisher gängige These von tiefgreifenden Modernisierungsprozessen zur Öffnung der Archive im 19. Jahrhundert, negiert insbesondere diejenige von Archivreformen und Öffnungen Anfang des 19. Jahrhunderts als Folgen der Französischen Revolution und der anschließenden Neuordnungen in Europa. Demgegenüber arbeitet Müller den langen Nachhall der frühneuzeitlichen Archivpolitik heraus. Die Untersuchung kreist vor allem um die Koordinaten von ,,arcana imperii“, ,,arcanum“/,secretum“ beziehungsweise arkanpolitischer Zensur in einer rechtspolitisch institutionellen Kultur einerseits und

K. Minner $(\bowtie)$

Universität Siegen, Siegen, Deutschland

E-Mail: katrin.minner@uni-siegen.de 
dem „historischen Imperativ“ der Forscher und Gelehrten zur persönlichen Inaugenscheinnahme als neuem Ausweis der Wissenschaftlichkeit andererseits.

Archiv- und Historiografiegeschichte sind - wie auch Müller in der Einleitung konzediert - keine neuen Themen oder Herangehensweisen. Die innovative Idee der Studie besteht darin, die auf Archive gestützte geschichtswissenschaftliche Forschung selbst zu historisieren, indem sie die Folgewirkungen archivpolitischer Rahmenbedingungen auf die Generierung historischen Wissens bemisst. Verdienst des Autors ist es, deutlich zu machen, wie erst im Laufe des 19. Jahrhunderts das persönliche Studium von Archivquellen zum Qualitätsmerkmal wissenschaftlicher Forschung und das Archiv damit zu einer wissenskonstitutiven Einrichtung wurde. Die Quellen wurden mit einer Vorstellung von „Reinheit“, ,Unberührtheit“ und „Wahrheit“" aufgeladen. Doch begründet er schlüssig, dass die daraus verfertigten Darstellungen keinesfalls den Anspruch erfüllen konnten, dass alle vorhandenen und relevanten Quellenbestände voraussetzungslos, frei, umfassend, ungestört oder gar wiederholt bearbeitet werden konnten. Ein Aufschwung ,vaterländischer“ Geschichtsschreibung ging nicht unbedingt mit einer engen und harmonischen Zusammenarbeit von Staat/Administration/Archiv auf der einen und Forschern/Gelehrten auf der anderen Seite einher.

Die Studie besteht aus drei Abschnitten. Logisch stringent beleuchtet sie zunächst die Institution Archiv als Ausgangspunkt, zeichnet dann im umfassendsten Teil (etwa 240 Seiten) die sukzessiven Entwicklungen zur Öffnung für eine wissenschaftliche Nutzung nach und bringt schließlich die gelehrte Wissensproduktion mit dem Archiv und seinen Rahmenbedingungen zusammen. Die Arbeit basiert auf der Untersuchung staatlicher Zentralarchive in Preußen und Bayern - an manchen Stellen mit Exkursen ins österreichische Hauptstaatsarchiv - und berücksichtigt dazu eine beeindruckende Zahl archivalischer Quellen.

Der erste Abschnitt („Die Archive“) bietet zum Teil bereits bekannte institutionsgeschichtliche Details zu den Bedingungen der Einsichtnahme. Archive blieben auch im 19. Jahrhundert Teil des Regierungshandelns und damit unter dessen materieller Verfügungsmacht und Organisationsprinzipien. Im zweiten Untersuchungsabschnitt (,Die Öffnung der Archive“) werden kleinteilig an vielen Einzelbeispielen Reformideen sowie Praktiken (vor allem Benutzungsgesuche beziehungsweise deren administrative Prüfung), Vorstöße und Rückschläge in der Entwicklung geschildert. Dies gipfelt in der Darstellung, wie die unterschiedlichen Haltungen zum Quellenzugang zwischen der Preußischen Akademie der Wissenschaften und den Archivaren und Ministerien beim Editionsprojekt der Schriften Friedrichs II. aufeinanderprallten und häufig erst durch den König entschieden wurden. Ein besonderes Augenmerk legt Müller auf die „Mikropolitik“ der Gelehrten, die sich mit Netzwerken, Lobbying und ,Self-Fashioning“ (S. 303) in eine möglichst günstige Ausgangssituation für ihre Gesuche zur Akteneinsicht zu bringen versuchten. Letztlich konstatiert der Autor, dass noch über die Mitte des 19. Jahrhunderts hinaus Reform- und Öffnungsideen sich nicht durchsetzten - in Preußen noch weniger als in Bayern - und der Wandel sich nur zögerlich und schwerfällig anließ. Der dritte Abschnitt, der eigentlich mit dem Thema des „Geschichte machens“ besonders spannend ist, sowie die Archivpolitik und deren Rückwirkungen auf die Forschung zusammenführt, kommt in der Studie mit etwa 40 Seiten allerdings knapp daher. Er oszilliert zwischen der 
neuartigen und zunehmenden Verflechtung von Forschung und Archiv und den weiterbestehenden Spannungen von Kontrolle und Begrenzung ohne festgeschriebenes und damit einforderbares Recht auf Akteneinsicht. Dies habe zu „Kompromissen“ (S. 375) in den wissenschaftlichen Darstellungen geführt, indem sich die Forscher zu einer Selbstzensur genötigt fühlten. Die tatsächlichen Wandlungen von einer Rechtstitel verwahrenden Institution zu einem sich professionalisierenden Archiv als Forschungseinrichtung ab der zweiten Hälfte des 19. Jahrhunderts werden nur kurz angerissen.

Als theoretisch-methodische Impulse greift die gekürzte Fassung der Göttinger Habilitationsschrift zwei Ideen auf: Zum einen rekurriert sie auf medienwissenschaftliche Ansätze, dass jeder „Produktion von Daten“ „(medien-)technische Voraussetzungen“" zugrunde liegen, die im späteren Werk kaschiert werden (S. 33). Dieser Ansatz wird mit dem Programm verwoben, Forschungspraxis (,doing science“ beziehungsweise „,doing history“) zu untersuchen (S. 34f.).

Die Arbeit beobachtet die Archivpolitik der beiden Länder Preußen und Bayern aus ihrer institutionellen Sicht und das Handeln der Forschenden. Je nach dem, was die bisweilen lückenhafte Quellenlage zu bieten hat, ,switcht' der Autor zwischen den beiden Ländern, sodass manchmal Fragen nach der tatsächlichen Vergleichbarkeit beziehungsweise der Verabsolutierung aufkommen. Etwas quer zum gewählten begrenzten räumlichen Zuschnitt liegen die Abschnitte zu den ,Höhenkamm-Gelehrten“ (zum Beispiel Leopold von Ranke), die auf Reisen im europäischen Ausland jenseits von Bayern und Preußen forschten und dort mit Hilfe von grenzüberschreitenden Netzwerken und anderen Einrichtungen, wie Bibliotheken, Wissen erarbeiteten.

Die Studie fokussiert mit ihrem Schwerpunkt der staatlichen Archivpolitik auf die zentralen landeseigenen Archive und ausgewiesene Spitzenforscher ihrer Zeit, was absolut legitim und nachvollziehbar ist. Doch kommen beim Lesen auch weitergehende Fragen auf, ob/wie diese Archivpolitik in die jeweiligen Staaten ausstrahlte. Wie sahen die Bedingungen zum Beispiel in den preußischen Provinzialarchiven aus? Standen diese unter den gleichen arkanpolitischen Vorgaben oder wandelten sich diese im Zuge einer „Gouvernementalität“ zu Schnittstellen mit regionalen Gelehrten und Geschichtsinteressierten vor Ort, die sich beispielsweise in Altertums-/ Historischen Vereinen sammelten und Urkundenbücher vorantrieben und damit auch Teil des politisch-gesellschaftlichen Kommunikationsprozesses wurden? Brachten nicht auch noch andere Akteure, wie beispielsweise der westfälische Oberpräsident Ludwig von Vincke, mit eigenen Ideen und einem gewissen regionalen ,Eigensinn ' weitere Impulse ein? Hier können noch andere Nuancen der Archivpolitik des 19. Jahrhunderts Aufschluss versprechen.

Spannend - aber vermutlich ein Quellenproblem - wären auch mehr Informationen gewesen, wieviel Wissen die Archivare selbst über die Inhalte der von ihnen gehüteten Quellen und die tagesaktuellen, politisch möglicherweise brisanten Entwicklungen tatsächlich hatten, um über eine Auslegungs-/Beurteilungskompetenz für die Vorlage - oder gar Zensur - von Materialien für die Forscher zu verfügen, beziehungsweise wie wiederum ihre Verschwiegenheit gesichert wurde. Am Rande werden ja einige Archivare genannt, die selbst Benutzungsgesuche für eigene Archivforschungen und wissenschaftlich-literarische Arbeiten vorlegten. Damit war 
die Trennung zwischen Archivar und Forscher vielleicht nicht so monolithisch, wie die Studie auf den ersten Blick glauben lässt.

Zusammenfassend lässt sich sagen, dass die Studie sich von der im Archivbereich verbreiteten rein institutionellen Sicht löst und ein gern und lange kolportiertes Narrativ von der einschneidenden Bedeutung der Revolution und Reformen am Anfang des 19. Jahrhunderts beziehungsweise dem Bedeutungsverlust des ,arcanum imperii“" zurecht kritisch hinterfragt und relativiert. Die Idee des Archivs als Ort oder auch Mittel der Geschichtsschreibung musste erst gegen Widerstände und Hindernisse verbreitet und sukzessive verwirklicht werden. Zwar ist es erklärtes Ziel des Verfassers, einen Wandel durch politisch-gesellschaftliche Kommunikationsprozesse nachzuvollziehen. Es entsteht dabei aber der Eindruck, dass die Modernisierungsschritte des 19. Jahrhunderts insgesamt (zu) gering geschätzt werden, was das 19. Jahrhundert doch etwas sehr als langen Abgesang der Frühen Neuzeit anmuten lässt. Für die Geschichte der Verknüpfung von Archiv und wissenschaftlicher Forschung leistet die Studie aber einen wichtigen Beitrag.

Funding Open Access funding enabled and organized by Projekt DEAL.

Open Access Dieser Artikel wird unter der Creative Commons Namensnennung 4.0 International Lizenz veröffentlicht, welche die Nutzung, Vervielfältigung, Bearbeitung, Verbreitung und Wiedergabe in jeglichem Medium und Format erlaubt, sofern Sie den/die ursprünglichen Autor(en) und die Quelle ordnungsgemäß nennen, einen Link zur Creative Commons Lizenz beifügen und angeben, ob Änderungen vorgenommen wurden.

Die in diesem Artikel enthaltenen Bilder und sonstiges Drittmaterial unterliegen ebenfalls der genannten Creative Commons Lizenz, sofern sich aus der Abbildungslegende nichts anderes ergibt. Sofern das betreffende Material nicht unter der genannten Creative Commons Lizenz steht und die betreffende Handlung nicht nach gesetzlichen Vorschriften erlaubt ist, ist für die oben aufgeführten Weiterverwendungen des Materials die Einwilligung des jeweiligen Rechteinhabers einzuholen.

Weitere Details zur Lizenz entnehmen Sie bitte der Lizenzinformation auf http://creativecommons.org/ licenses/by/4.0/deed.de. 